\title{
SIMULAÇÃO DE RESERVATÓRIO DE PETRÓLEO UTILIZANDO O MODELO BLACK-OIL EM CONJUNTO COM MALHAS NÃO-ESTRUTURADAS
}

\author{
R. L. Nogueira'; I. C. M. Lima ${ }^{2}$; B. R. B. Fernandes ${ }^{3}$ e F. Marcondes ${ }^{4}$ \\ ${ }^{1}$ Instituto Federal do Rio Grande do Norte - Câmpus Apodi; ${ }^{2,3}$ Laboratório de Dinâmica de Fluidos \\ Computacional - Universidade Federal do Ceará e ${ }^{4}$ Departamento de Engenharia Metalúrgica e Ciências dos \\ Materiais - Universidade Federal do Ceará. \\ regis.nogueira@ifrn.edu.br_marcondes@ufc.br
}

Artigo submetido em novembro/2011 e aceito em maio/2012

\section{RESUMO}

Dentre as várias metodologias disponíveis para solução numérica de escoamentos, o método EbFVM em conjunto com malhas não-estruturadas tem recebido grande atenção por parte da comunidade de simulação de reservatórios de petróleo. Nesta metodologia o domínio de cálculo é divido em elementos e cada elemento é dividido em sub-elementos de acordo com o número de vértices. Os volumes de controle são construídos em torno dos vértices dos elementos, originando uma metodologia centrada nos vértices da malha (cell vertex). Este trabalho apresenta uma investigação da metodologia EbFVM usando o modelo black-oil com base na pressão e frações mássicas globais.
Nesta abordagem, mesmo quando toda a fase gás encontra-se dissolvida na fase óleo à fração mássica global de gás será diferente de zero. Nesse trabalho, a abordagem previamente mencionada é aplicada a fluxos multifásicos envolvendo óleo, gás e água. As equações de balanço de massa em termos de fração mássica global de óleo, gás e água e pressão são aproximadas através da metodologia EbFVM, utilizando um procedimento totalmente implícito. Com o intuito de validar a formulação adotada neste trabalho serão também apresentados os resultados obtidos com o simulador IMEX da CMG em conjunto com malhas cartesianos para alguns dos casos testes selecionados.

PALAVRAS-CHAVE: modelo black-oil, frações mássicas globais, EbFVM, fluxo multifásico, malhas nãoestruturadas.

\section{UNSTRUCTURED GRIDS AND AN ELEMENT BASED CONSERVATIVE APPROACH FOR A BLACK-OIL RESERVOIR SIMULATION}

\begin{abstract}
From several methodologies available, the Element based Finite Volume Method (EbFVM), in conjunction with unstructured meshes, is a methodology that deserves large attention in the petroleum reservoir simulation. After the initial step of discretization, each element is divided into subelements and the mass balance for each component is developed for each sub-element (sub-control volumes). This work presents an investigation of an element-based approach using the black-oil model based on pressure and global mass fractions. In this approach, even when all gas phase is dissolved in oil phase the global mass fraction of gas will be different from zero. Therefore, no
\end{abstract}

additional numerical procedure is necessary in order to treat the gas phase appear/disappearance. In this work, the above mentioned approach is applied to multiphase flows involving oil, gas, and water. The mass balance equations in terms of global mass fraction of oil, water and global mass fractions are discretized through the EbFVM using a fully implicit procedure. In order to validate the numerical results of the proposed methodology, the results obtained with the IMEX simulator (Implicit-Explicit Black Oil Simulation) from CMG (Computer Modeling Group) are presented for some of the chosen case studies.

KEY-WORDS: Black-oil model, Global mass fractions, EbFVM, Multiphase flow, Unstructured meshes. 


\section{SIMULAÇÃO DE RESERVATÓRIO DE PETRÓLEO UTILIZANDO O MODELO BLACK-OIL EM CONJUNTO COM MALHAS NÃO-ESTRUTURADAS}

\section{INTRODUÇÃO}

As malhas não-estruturadas são ferramentas importantes para a modelagem de importantes características dos reservatórios, tais como fraturas discretas, falhas geológicas, fronteiras irregulares e poços desviados. Das diversas opções de malhas não-estruturadas disponíveis, o EbFVM (Element based Finite-Volume Method) é uma metodologia que tem recebido muita atenção (Cordazzo, 2004; Cordazzo et al., 2004a, b; Araújo, 2004; Marcondes e Sepehrnoori, 2007, Karpinski et al., 2009; Marcondes e Sepehrnoori, 2010; Nogueira et al., 2010), principalmente pelo fato da metodologia EbFVM ser localmente conservativa.

Uma maneira de obter os volumes de controle não-estruturados é através do método das medianas. Neste caso, cada elemento é dividido em subelementos ligando-se o centroide do elemento com o segmento médio de cada segmento do elemento. Este processo de obtenção dos volumes é empregado no modelo numérico desenvolvido neste trabalho, e é geralmente encontrado na literatura de volumes finitos utilizando malhas não-estruturadas (Baliga e Patankar, 1988). Este trabalho propõe uma metodologia para simulação numérica de escoamentos multifásicos em reservatórios de petróleo usando o método dos volumes finitos baseado em Elementos Finitos (EbFVM). Este método emprega elementos triangulares ou quadrangulares para a discretização bidimensional, o que lhe confere maior flexibilidade e generalidade.

A principal contribuição do presente trabalho é a abordagem do problema trifásico, multicomponente para a simulação de processos de recuperação primária e secundária através da injeção de água ou gás utilizando o modelo black-oil nas frações mássicas aliado ao emprego de malhas não-estruturadas bidimensionais.

\section{MATERIAIS E MÉTODOS}

O modelo black-oil é comumente escrito em termos das pressões e saturações das fases. No entanto, esta formulação tem problemas de implementação numérica quando o componente gás encontra-se totalmente miscível com a fase óleo. Neste caso, é necessária uma mudança de variável ou a utilização de valores pequenos da saturação de gás durante a simulação para se evitar a mudança de variável. Com o intuito de se evitar o emprego de artifícios numéricos para a implementação do modelo black-oil quando o componente gás encontra-se totalmente dissolvido com a fase óleo Prais e Campagnolo (1991) propuseram que o modelo black-oil fosse escrito em termos de frações mássicas dos componentes e pressões das fases. Cunha (1996) utilizou esta formulação para a solução do escoamento bifásico (óleoágua) aliado com o emprego de coordenadas generalizas tridimensionais. Os termos cruzados oriundos da discretização foram mantidos no lado direito do sistema linear com o intuito de diminuir o sistema linear. Coutinho (2002) utilizou esta formulação para a solução de problemas bidimensionais envolvendo o escoamento óleo-água utilizando coordenadas generalizadas. Neste trabalho foi realizada uma investigação sobre a importância de se manter os termos cruzados oriundos da discretização no sistema linear. Foi verificado que caso a malha 
seja quase ortogonal, podem-se manter os termos não-ortogonais no lado direito do sistema linear. Destaca-se que na maioria dos casos é quase impossível respeitar tal condição. 0 trabalho de Coutinho (1996) deu origem ao simulador BLACK. Este simulador foi escrito em linguagem $\mathrm{C}++$ orientado a objetos. Araújo (2004) introduziu coordenadas generalizadas tridimensionais (3D) no simulador BLACK para a solução do escoamento óleo-água e realizou a implementação de um procedimento de inicialização de variáveis para permitir a segregação das fases. Sarmento (2009) resolveu o problema trifásico (óleo, gás, água) em conjunto com coordenadas generalizadas tridimensionais. O presente trabalho inclui a formulação EbFVM (Element based Finite-Volume Method) no simulador BLACK para a solução de problemas trifásicos em geometrias bidimensionais em conjunto com o modelo black-oil em termos das frações mássicas e pressões. Esta formulação tem sido largamente empregada na literatura (Cordazzo, 2004; Cordazzo et al, 2004a, b; Araújo, 2005; Marcondes e Sepehrnoori, 2007, Karpinski et al. 2009; Marcondes e Sepehrnoori, 2010; Nogueira et al., 2010; Nogueira, 2011).

\subsection{MODELO MATEMÁTICO}

A seguir serão apresentadas a formulação do modelo composicional e as simplificações requeridas para obtenção do modelo black-oil para os três componentes (água, óleo e gás) em termos das frações mássicas e pressão que será utilizada no presente trabalho. São também apresentadas às equações de restrições para o modelo black-oil em termos de pressões capilares e restrição mássica necessárias para o fechamento do sistema de equações.

Assumindo que existam no reservatório $n_{p}$ fases e $n_{c}$ componentes hidrocarbonetos distribuídos entre as fases, pode-se obter a equação de conservação de massa para cada componente no meio poroso (reservatório) em termos de saturações ou frações mássicas. Destaca-se que, devido à transferência de massa de cada componente entre as fases, a massa que se conserva é a do componente e não a da fase. A equação de conservação de massa para cada componente pode ser escrita em termos das saturações como,

$$
\frac{\partial}{\partial t}\left[\phi\left(\rho_{p} S_{p} X_{i p}\right)\right]=-\nabla \cdot\left(\sum_{n p} \rho_{p} X_{i p} u_{p}^{\text {um }}\right)-\sum_{n p} X_{i p} \bar{m}_{p}
$$

A [Eq. 01] representa a equação de conservação do componente $i$ para o modelo composicional em termos das saturações e pressões. O termo do lado esquerdo da [Eq. 01] representa a taxa de decréscimo/acúmulo de massa do componente $i$ por unidade de volume do reservatório (conhecido como o termo de acumulação). O primeiro termo no lado direito representa o transporte advectivo do componente $i$ na fase $p$ e o último termo no lado direito denota o termo fonte/sumidouro do componente $i$ ocasionado pela produção/injeção da fase $p$ nos poços.

Na [Eq. 01], $\bar{m}_{p}$ representa a vazão mássica da fase $p$ por unidade de volume poroso do bloco contendo o poço. Este termo é igual ao produto da densidade da fase $p\left(\rho_{p}\right)$ pela vazão volumétrica da fase $\mathrm{p}$ por unidade de volume poroso do bloco contendo o poço $\left(q_{p}\right)$. O termo $q_{p}$ é positivo para poços de injeção e negativo para poços de produção. Deve ser observado que 
na [Eq. 01] foram desprezados os termos de transporte do componente $i$ por difusão. Esta hipótese é comumente adotada para processos de recuperação de petróleo que não envolva alteração da viscosidade do fluido injetado através da injeção de solventes, polímeros, etc. A velocidade da fase $p, u_{p}$, é dada pela lei de Darcy,

$$
\text { unt } u_{p}=-\lambda_{p} \overline{\bar{K}} \cdot \nabla \Phi_{p}
$$

Onde $\Phi_{p}$ é o potencial da fase $p$, definido como:

$$
\Phi_{p}=p_{p}+\rho_{p} g Z
$$

Onde $p_{p}$ é a pressão da fase $p, g$ é a aceleração da gravidade e $Z$ é a coordenada vertical, sendo positiva no sentido contrário ao vetor gravitacional.

Substituindo a [Eq. 02] na [Eq. 01], obtém-se:

$$
\frac{\partial}{\partial t}\left[\phi\left(\rho_{p} S_{p} X_{i p}\right)\right]=\nabla \cdot\left(\sum_{n p} \rho_{p} X_{i p} \lambda_{p} \overline{\bar{K}} . \nabla \Phi_{p}\right)-\sum_{n p} X_{i p} \bar{m}_{p}
$$

Utilizando o conceito de frações mássicas globais, a [Eq. 04] pode ser reescrita em termos de pressão e frações mássicas globais. Desta forma o termo de acúmulo pode ser escrito como:

$$
\frac{\partial}{\partial t}\left[\phi\left(\sum_{n p} \rho_{p} S_{p} X_{i p}\right)\right]=\frac{\partial}{\partial t}\left[\phi \rho_{m} Z_{i}\right]
$$

Substituindo-se a [Eq. 05] na [Eq. 04] obtém-se a equação do modelo composicional em termos de frações mássicas globais e pressões.

$$
\frac{\partial}{\partial t}\left[\phi \rho_{m} Z_{i}\right]=\nabla \cdot\left(\sum_{n p} \rho_{p} X_{i p} \lambda_{p} \overline{\bar{K}} \cdot \nabla \Phi_{p}\right)-\sum_{n p} X_{i p} \bar{m}_{p}
$$

O modelo black-oil é uma simplificação do modelo composicional e adequa-se a reservatórios contendo óleos pesados ou de baixa volatilidade. Desta forma, este modelo tem a característica de ser, em geral, um modelo isotérmico cujo comportamento das fases é regido por relações PVT (pressão, volume e temperatura). No modelo black-oil adotado no presente trabalho, apenas o componente gasoso pode estar miscível totalmente ou parcialmente na fase óleo. Não existe transferência de massa entre as fases óleo e água e os componentes óleo e água não vaporizam na fase gás.

Para formular matematicamente o modelo black-oil, normalmente utiliza-se como variáveis primárias a pressão do óleo e as saturações de cada fase. Todavia, este tipo de formulação apresenta sérios problemas em escoamentos envolvendo a fase gasosa. Isto ocorre porque, em algumas regiões do reservatório, devido às condições termodinâmicas, a fase gás pode desaparecer (componente gás completamente dissolvido na fase óleo). Nestas condições, a equação da fase gás torna-se irrelevante (Abou-Kassem e Aziz, 1985) e (Forsyth e Sammon, 
1984). Visando contornar estas dificuldades, desenvolveu-se a formulação que utiliza as frações mássicas globais de cada componente no lugar das saturações das fases (Prais e Campagnolo, 1991; Cunha, 1996; Maliska et al., 1997).

De posse das frações mássicas dos componentes água, óleo e gás na fase água ( $X_{w w}$, $\left.X_{o w}, X_{g w}\right)$ obtém-se as equações do modelo black-oil para os componentes água, óleo, gás em termos de frações mássicas globais e pressão de cada fase. Estas equações são dadas por:

$$
\begin{aligned}
& \frac{\partial}{\partial t}\left(\phi \rho_{m} Z_{w}\right)=\nabla \cdot\left(\lambda_{w} \overline{\bar{K}} \cdot \nabla \Phi_{w}\right)-\bar{m}_{w} \\
& \frac{\partial}{\partial t}\left(\phi \rho_{m} Z_{o}\right)=\nabla \cdot\left(X_{o o} \lambda_{o} \overline{\bar{K}} \cdot \nabla \Phi_{o}\right)-X_{o o} \bar{m}_{o} \\
& \frac{\partial}{\partial t}\left(\phi \rho_{m} Z_{g}\right)=\nabla \cdot\left[\left(1-X_{o o}\right) \lambda_{o} \overline{\bar{K}} \cdot \nabla \Phi_{o}+\lambda_{g} \overline{\bar{K}} \cdot \nabla \Phi_{g}\right]-\left(1-X_{o o}\right) \bar{m}_{o}-\bar{m}_{g}
\end{aligned}
$$

Somando as [Eq. 07]-[Eq. 09] obtém-se a seguinte equação de conservação global da de massa:

$$
\frac{\partial}{\partial t}\left(\phi \rho_{m}\right)=\nabla \cdot\left[\lambda_{w} \overline{\bar{K}} \cdot \nabla \Phi_{w}+\lambda_{o} \overline{\bar{K}} \cdot \nabla \Phi_{o}+\lambda_{g} \overline{\bar{K}} \cdot \nabla \Phi_{g}\right]-\bar{m}_{w}-\bar{m}_{o}-\bar{m}_{g}
$$

Nas [Eq. 07]-[Eq. 09], $\phi$ denota a porosidade, $\rho_{m}$ densidade média, $Z_{w}, Z_{o}, Z_{g}$ são, respectivamente, as frações globais de massa de água, óleo e gas; $X_{o o}$ a fração mássica do componente óleo em fase óleo; $\lambda_{w}, \lambda_{o}, \lambda_{g}$ são, respectivamente, a mobilidade de água, óleo e gás, $(\overline{\bar{K}})$ é o tensor de permeabilidade absoluta.

Das [Eq. 07]-[Eq. 09] pode-se verificar que há seis incógnitas $\left(Z_{w}, Z_{o}, Z_{g}, P_{w}, P_{o}\right.$, e $\left.P_{g}\right)$ e apenas três equações, uma vez que a [Eq. 10] é apenas uma combinação das [Eq. 07]-[Eq. 09]. As equações de fechamento vêm de relações experimentais (pressões capilares) e balanço de massa. Desta forma, as equações que completam o modelo matemático são dadas por,

$$
\begin{aligned}
& P_{c o g}=P_{g}-P_{o} \\
& P_{c o w}=P_{o}-P_{w} \\
& Z_{w}+Z_{O}+Z_{g}=1
\end{aligned}
$$

13]

As condições de contorno utilizadas no presente trabalho, para o escoamento de cada fase, se referem a fronteiras impermeáveis e são dadas por:

$$
\overline{\bar{K}} \cdot \nabla \Phi_{p} \cdot d \stackrel{\text { ut }}{S}=0
$$

Diferentemente do método dos volumes finitos tradicional, muitas vezes chamado de método de diferenças finitas na literatura do petróleo, onde os elementos e volumes de controle são coincidentes (construção de células centradas - cell center), na metodologia EbFVM os volumes de controle são construídos em torno dos vértices dos elementos, originando uma metodologia centrada nos vértices da malha (cell vertex). Nesta contrução, 
cada elemento é dividido em subelementos ligando-se o centróide geométrico de cada elemento com o segmento médio de cada elemento. O volume de controle resultante é formado pelas porções (sub-volume de controle - Sc) de elementos vizinhos, como mostrado na figura 1.

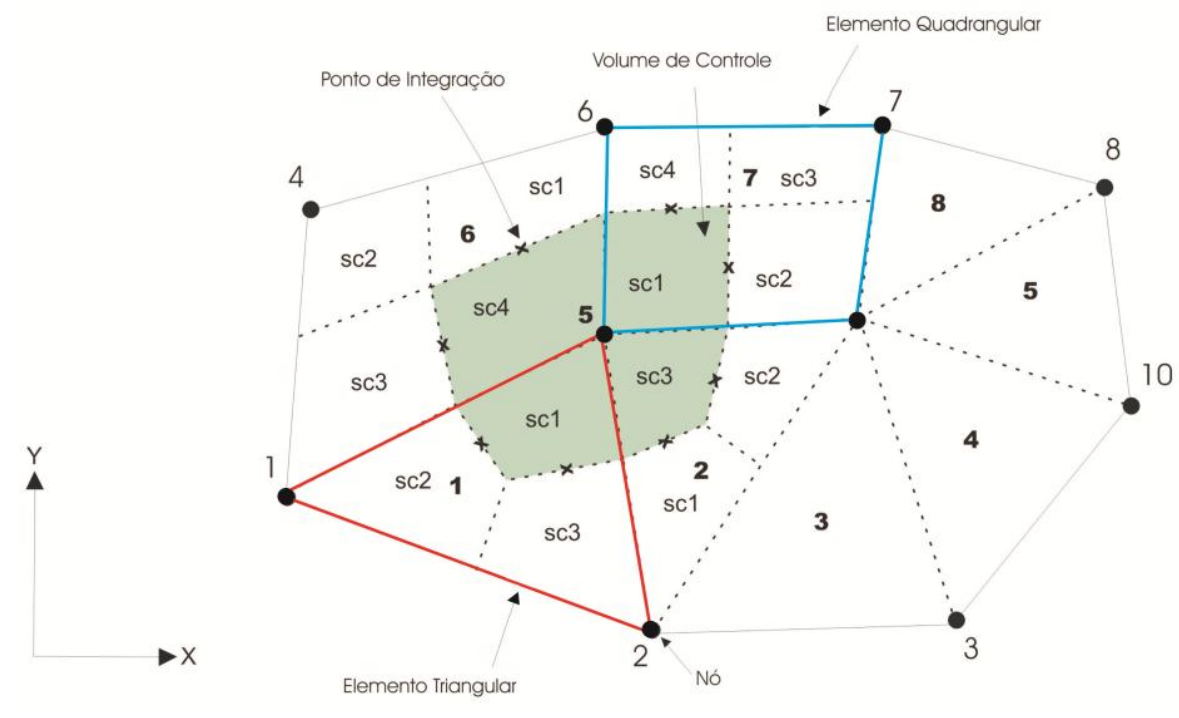

Figura 1 - Volume de controle.

Neste trabalho as [Eq. 07]-[Eq. 10] são usadas para avaliar $Z_{w}, Z_{o}$, e $p_{o}$. A fim de lidar com as características complexas dos reservatórios, como limites irregulares. Conforme descrito em Marcondes e Sepehrnoori (2010), na metodologia EbFVM cada elemento é dividido em sub-elementos. No restante do presente trabalho estes subelementos serão subvolumes de controle. A figura 2 apresenta os volumes de controle para o elemento triangular e quadrangular, respectivamente. Deve ser observado que em cada subvolume de controle existem dois pontos de integração onde os termos advectivos devem ser avaliados. As equações de conservação para os componentes óleo e água e a equação de conservação global de massa [Eq. 07]-[Eq. 09] devem ser integradas para cada um dos subvolumes de controle.

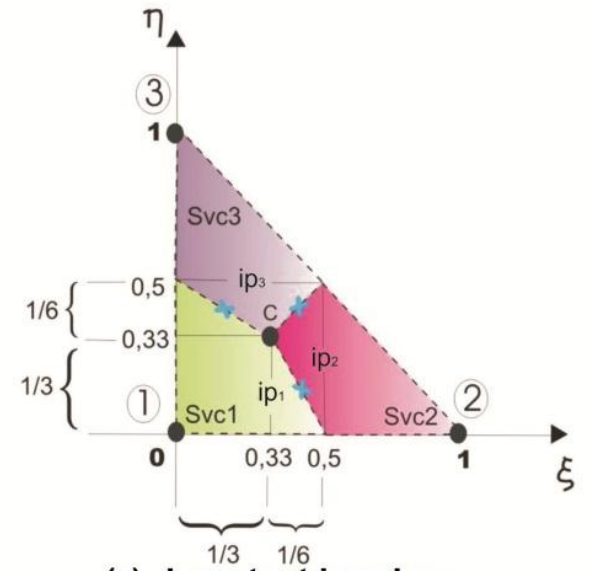

(a) elementos triangulares

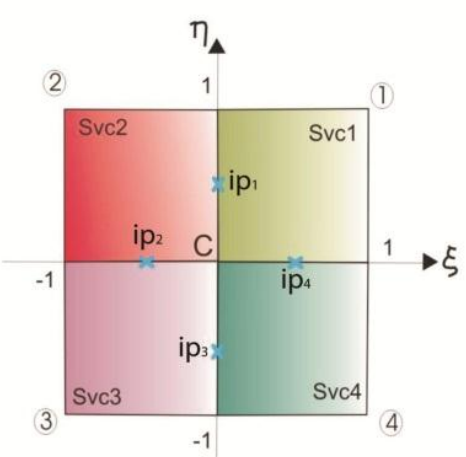

(b) elementos quadrangulares

Figura 2 - Elementos triangulares e quadrangulares. 
Integrando-se a equação da componente água no tempo e em cada um dos subvolumes de controle apresentados na Figura 2, empregando-se o teorema da divergência para os temos advectivos e avaliando-se as mobilidades e termos fonte/sumidouro através de um procedimento totalmente implícito e dividindo-se cada termo do lado direito pelo passo de tempo $(\Delta t)$, obtém-se:

$$
\int_{V}^{[}\left[\frac{\left(\phi \rho_{m} Z_{w}\right)-\left(\phi \rho_{m} Z_{w}\right)^{0}}{\Delta t}\right] d V=\int_{S} \lambda_{p} \overline{\bar{K}} \cdot \nabla \Phi_{w} d \stackrel{\mathbb{u}}{S}-\int_{V} \bar{m}_{w} d V
$$

Para avaliar os gradientes, é necessário obter as derivadas das funções de forma em relação à $x$ e $y$. Essas derivadas são dadas por:

$$
\begin{aligned}
& \frac{\partial N_{i}}{\partial x}=\frac{1}{\operatorname{det}\left(J_{t}\right)}\left(\frac{\partial N_{i}}{\partial \xi} \frac{\partial y}{\partial \eta}-\frac{\partial N_{i}}{\partial \eta} \frac{\partial y}{\partial \xi}\right) \\
& \frac{\partial N_{i}}{\partial y}=\frac{1}{\operatorname{det}\left(J_{t}\right)}\left(\frac{\partial N_{i}}{\partial \eta} \frac{\partial x}{\partial \xi}-\frac{\partial N_{i}}{\partial \xi} \frac{\partial x}{\partial \eta}\right)
\end{aligned}
$$

Onde $J_{t}$ é o Jacobiano da transformação dada por:

$$
\operatorname{det}\left(J_{t}\right)=\left(\frac{\partial x}{\partial \xi} \frac{\partial y}{\partial \eta}-\frac{\partial x}{\partial \eta} \frac{\partial y}{\partial \xi}\right)
$$

Usando uma aproximação do ponto médio ao longo de cada ponto de integração na [Eq. 15], resulta na seguinte equação para cada subvolume de controle:

$$
\begin{aligned}
& \left(\left(\frac{\phi \rho_{m} Z_{w}}{\Delta t}\right)_{i}-\left(\frac{\phi \rho_{m} Z_{w}}{\Delta t}\right)_{i}^{0}\right) V_{S C V_{i}}= \\
& \lambda_{w, i p 1}\left[\left(K_{11} \frac{\partial \Phi_{w}}{\partial x}+K_{12} \frac{\partial \Phi_{w}}{\partial y}\right) \Delta S_{1}+\left(K_{12} \frac{\partial \Phi_{w}}{\partial x}+K_{22} \frac{\partial \Phi_{w}}{\partial y}\right) \Delta S_{2}+\right]_{i p 1}+ \\
& \lambda_{w, i p 2}\left[\left(K_{11} \frac{\partial \Phi_{w}}{\partial x}+K_{12} \frac{\partial \Phi_{w}}{\partial y}\right) \Delta S_{1}+\left(K_{12} \frac{\partial \Phi_{w}}{\partial x}+K_{22} \frac{\partial \Phi_{w}}{\partial y}\right) \Delta S_{2}+\right]_{i p 2}-\bar{m}_{w} \\
& ; i=1, N N E
\end{aligned}
$$

Pode-se observar da [Eq. 19] que as mobilidades e gradientes de potenciais são avaliados no instante de tempo $t+\Delta t$. Deve ser observado ainda que a [Eq. 19] denota a conservação de massa do componente aquoso para cada um dos subvolumes de controle. A equação de conservação do volume de controle será obtida através do somatório de todos os subvolumes de controle que compartilham o mesmo vértice. Com o intuito de simplificação representar-se-á a [Eq. 19] da seguinte forma:

$$
A_{C C_{w, i}}+F_{w, i}+m_{i}=0 ; i=1, N N E
$$

Onde $A C C_{w, i}, F_{w, i}$ e $m_{i}$ denotam respectivamente as expressões dos termos de acumulação, advectivo e termo fonte ou sumidouro. As expressões dos termos de acumulação e termo fonte ou sumidouro são dados por: 


$$
\begin{aligned}
& A_{C C_{w, i}}=\left(\left(\frac{\phi \rho_{m} Z_{w}}{\Delta t}\right)_{i}-\left(\frac{\phi \rho_{m} Z_{w}}{\Delta t}\right)_{i}^{0}\right) V_{S C V_{i}} ; i=1, N N E \\
& F_{w, i}=\lambda_{w, i p 1}\left[\left(K_{11} \frac{\partial \Phi_{w}}{\partial x}+K_{12} \frac{\partial \Phi_{w}}{\partial y}\right) \Delta S_{1}+\left(K_{12} \frac{\partial \Phi_{w}}{\partial x}+K_{22} \frac{\partial \Phi_{w}}{\partial y}\right) \Delta S_{2}+\right]_{i p 1}+ \\
& \lambda_{w, i p 2}\left[\left(K_{11} \frac{\partial \Phi_{w}}{\partial x}+K_{12} \frac{\partial \Phi_{w}}{\partial y}\right) \Delta S_{1}+\left(K_{12} \frac{\partial \Phi_{w}}{\partial x}+K_{22} \frac{\partial \Phi_{w}}{\partial y}\right) \Delta S_{2}+\right]_{i p 2}-\bar{m}_{w} \\
& ; i=1, N N E
\end{aligned}
$$

Ao se inspecionar a [Eq. 22], pode-se inferir que é necessário avaliar as densidades, fração mássica, e mobilidades em duas interfaces de cada subvolume de controle. Para avaliar essas propriedades, um esquema upwind será usado conforme descrito em Cordazzo (2004).

A [Eq. 22] denota a conservação de cada subvolume de controle de cada elemento. Agora, é necessário montar a equação de cada volume de controle, obtida pela contribuição de cada subvolume de controle que compartilha o mesmo vértice. Este processo é semelhante à montagem da matriz de rigidez global no método dos elementos finitos (HUGHES, 1987). A figura 1 apresenta um volume de controle ao redor do vértice 5 . Este volume de controle receberá contribuições dos seguintes subvolumes de controles (SC): Sc1 do elemento 1, Sc3 do elemento 2, Sc4 do elemento 6 e Sc1 do elemento 7. Finalmente, destaca-se que na formulação adotada neste trabalho cada elemento tem um tensor permeabilidade absoluta e uma porosidade constante. No entanto, pode existir variação destas propriedade aos longo do domínio de cálculo (elementos).

As condições de contorno utilizadas em todos os problemas considerados resumem-se à fronteira impermeável do reservatório, vazões de gás ou água prescrita nos poços de injeção, e pressão de fundo de poço prescrita nos poços de produção. Com o intuito de validar a formulação adotada neste trabalho serão também apresentados os resultados obtidos com o simulador IMEX da CMG (Computer Modelling Group) em conjunto com malhas cartesianas para alguns dos casos-testes selecionados.

\section{RESULTADOS E CONCLUSÕES}

Baseado em diversos testes realizados foram adotados os seguintes valores para checar a convergência do processo de linearização de Newton em cada passo de tempo: $1,0 \times 10^{-9}$ para as frações mássicas globais do óleo e da água e 68947,57 Pa (10 psi) para a pressão do óleo. Testes com valores menores para as frações mássicas e pressão do óleo resultaram em resultados idênticos em termos de curvas de produção. Os sistemas lineares em cada passo de tempo foram resolvidos através do método Bi-CGSTAB (Method of Bi-Conjugate Gradient Stabilized), van der Vorst (1992) pré-condicionado à direita com uma fatoração incompleta de ordem 1 (ILU(1)), de acordo com Marcondes et al. (1995).

A fim de mostrar a aplicação da metodologia empregada no presente trabalho, dois casos testes foram selecionados. O primeiro caso refere-se à simulação do processo de recuperação secundária através da injeção de gás e água em um quarto da configuração fivespot (4 poços produtores e um poço injetor). Resultados obtidos com o simulador IMEX serão 
também apresentados. A Tabela 1 apresenta as propriedades físicas dos fluidos, bem como os dados do reservatório.

Tabela 1 - Dados do reservatório e dos fluidos.

\begin{tabular}{l|l|l|l}
\hline \multicolumn{1}{c|}{ Dados do Reservatório } & \multicolumn{1}{c|}{ Condições Iniciais } & Propriedades Físicas & Condições do poço \\
\hline$K=9.9 \times 10^{-13} \mathrm{~m}^{2}$ & $P=20 \times 10^{6} \mathrm{~Pa}$ & $B_{w}=1 \mathrm{e} 0 \mathrm{~Pa}$ & $P_{w f}=20 \times 10^{6} \mathrm{~Pa}$ \\
$A=2.1404 \times 10^{6} \mathrm{~m}^{2}$ & $S_{w i}=0.12$ & $c_{w}=1.00 \times 10^{-10} \mathrm{~Pa}^{-1}$ & $q_{i}=17280 \mathrm{~m} / \mathrm{d}$ \\
$h=15 \mathrm{~m}$ & $S_{o i}=0.88$ & $c_{o}=1.985 \times 10^{-9} \mathrm{~Pa}^{-1}$ & \\
$\phi=0.2$ & & & \\
$c_{r}=0.0$ & & & \\
$r_{w}=0.122 \mathrm{~m}$ & & & \\
\hline
\end{tabular}

Conforme pode ser observado na Tabela 1, a condição inicial do reservatório corresponde a um reservatório subsaturado, visto que existem no reservatório apenas água e óleo. Neste caso, com a injeção de gás no poço de injeção deve surgir naturalmente gás livre no interior do reservatório durante o processo de injeção. Por outro lado, com a injeção de água deverá existir no reservatório apenas água e óleo, visto que a pressão no interior do reservatório deverá ser sempre superior à pressão de bolha.

As Figuras 3 e 4 apresentam os campos de saturação de gás para o caso com injeção de gás em 145 dias de produção, tempo no qual é observada a chegada da frente de gás ao poço de produção. Apesar das diferenças significativas das malhas empregadas pode ser observada uma excelente concordância entre as frentes de gás obtidas. Destaca-se que a maior suavização das frentes obtidas com as malhas de quadriláteros e triângulos deve-se unicamente ao processo de visualização empregado - Kraken da ESSS (Engineering Simulation Scientific Software). Para as malhas não estruturadas as propriedades são armazenadas nos vértices da malha e desta forma um processo de interpolação é realizado no momento de visualizar os resultados. Para a malha cartesiana, cada propriedade armazenada no centro de cada volume é pintada com uma cor de acordo com o valor desta propriedade.
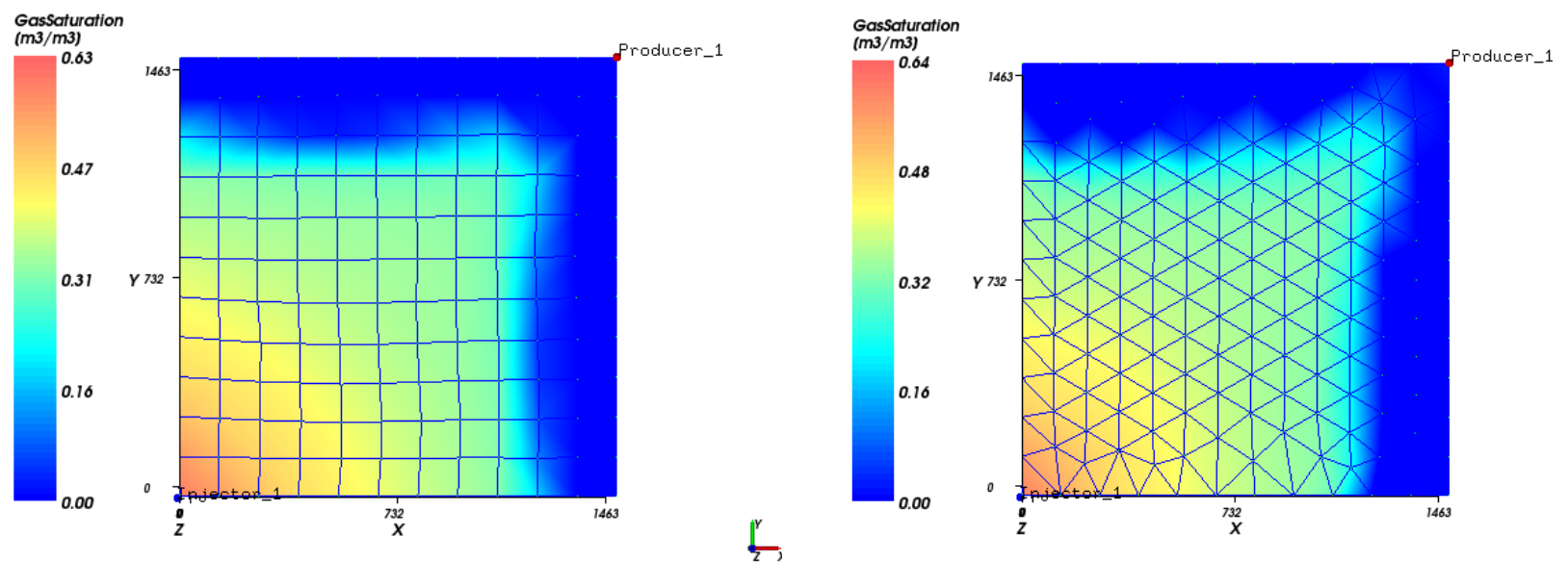

Figura 3 - Saturação de gás - 145 dias - malha quadrangular e malha triangular. 


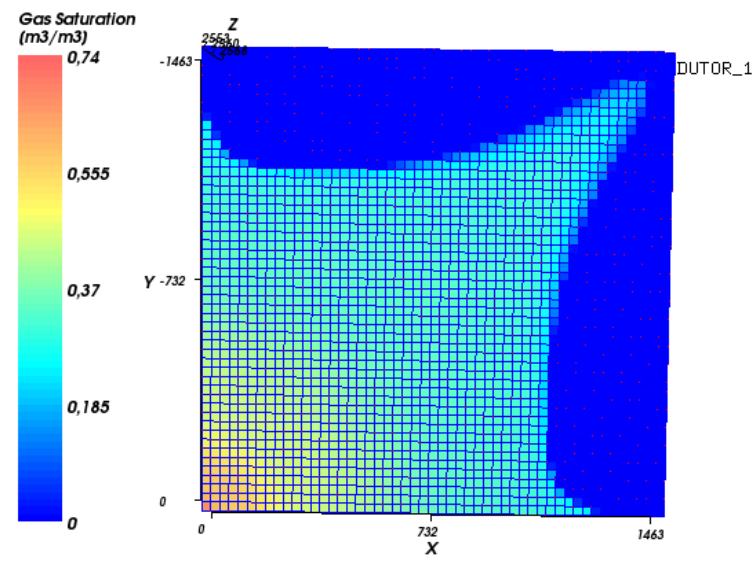

Figura 4 -Saturação de gás - 145 dias - IMEX 51x51.

Os campos de saturação de água para o caso 1 em 145 dias de produção, tempo no qual é observada a chegada da frente de água ao poço de produção, considerando a injeção de água ao invés de gás estão listados nas Figuras 5 e 6 . As frentes de saturação de água obtidas com a metodologia EbFVM apresentaram também uma boa concordância com a frente de água obtida com o simulador IMEX. Outro ponto importante a ser destacado é que mesmo não existindo gás livre no reservatório a formulação black-oil nas frações mássicas resolveu o caso com injeção de água sem nenhum tratamento numérico adicional.
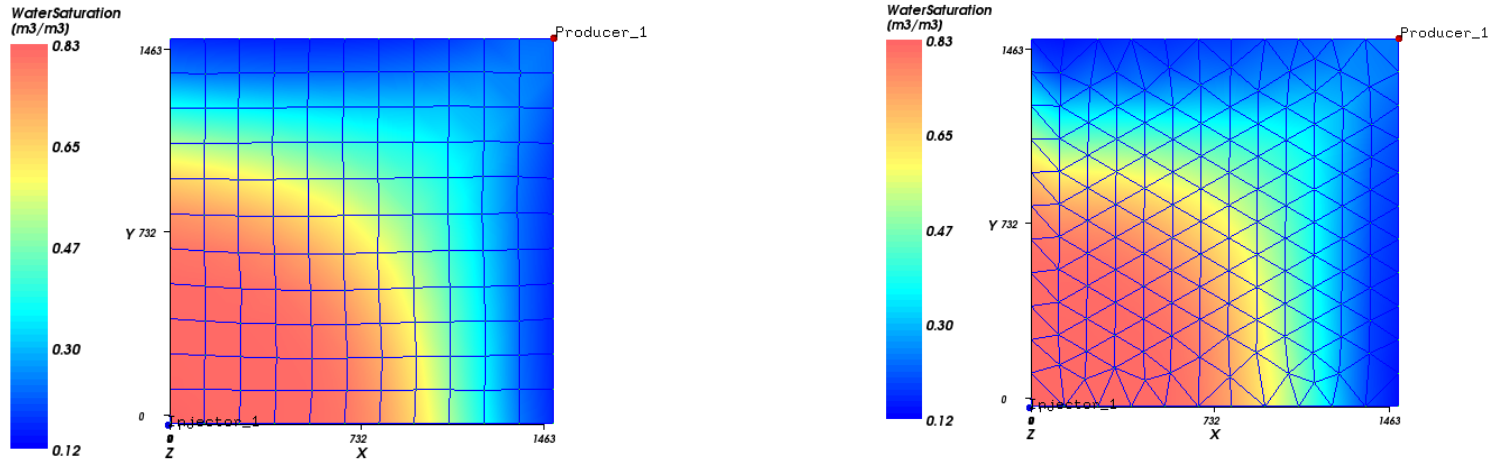

Figura 5 - Saturação de água - 145 dias - malha quadrangular e malha triangular.

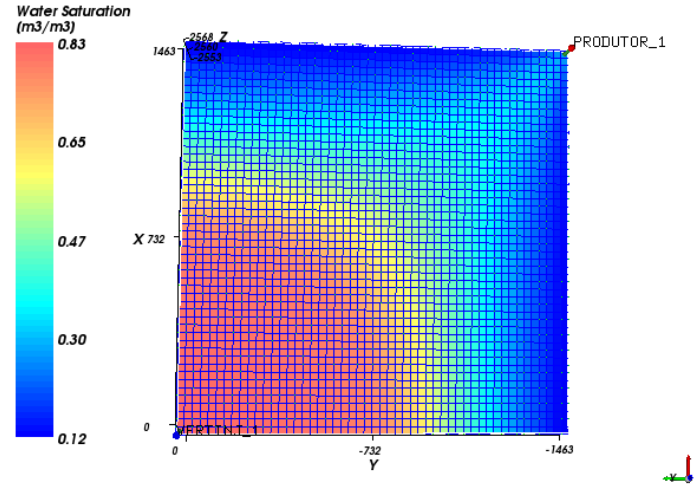

Figura 6 - Saturação de água - 145 dias - IMEX 51x51. 
Por outro lado, para as vazões de gás, diferenças mais significativas entres os resultados dos dois simuladores foram observadas. As diferenças verificadas entre os resultados obtidos com a formulação da presente dissertação e os resultados do IMEX podem ser atribuídas à localização dos poços nos dois simuladores, cálculo das densidades da fase gás e a diferente formulação para o cálculo das variáveis. No simulador IMEX, cada poço é localizado no centro de cada volume de controle, enquanto que na presente formulação cada poço é localizado nos vértices da malha. Isto aumenta a distância entre produtor injetor para o presente caso quando comparado com o simulador IMEX. Com relação à densidade da fase gasosa, a explicação para tal comportamento pode ser buscada na maior compressibilidade do gás quando comparado com aquela das curvas do óleo e da água e da dificuldade de se utilizar exatamente as mesmas propriedades nos dois códigos utilizados. Finalmente, destaca-se que as incógnitas no simulador utilizado neste trabalho são as frações mássicas ao passo que no IMEX são as saturações. As Figuras 7 e 8 apresentam as curvas de volume de óleo e gás acumulados, respectivamente. Apesar das diferenças observadas em termos das vazões para este caso, as diferenças em termos de volumes acumulados foram menores. A razão para tal comportamento é justificada pelo fato da ocorrência da interseção dos gráficos obtidos com ambos os simuladores.
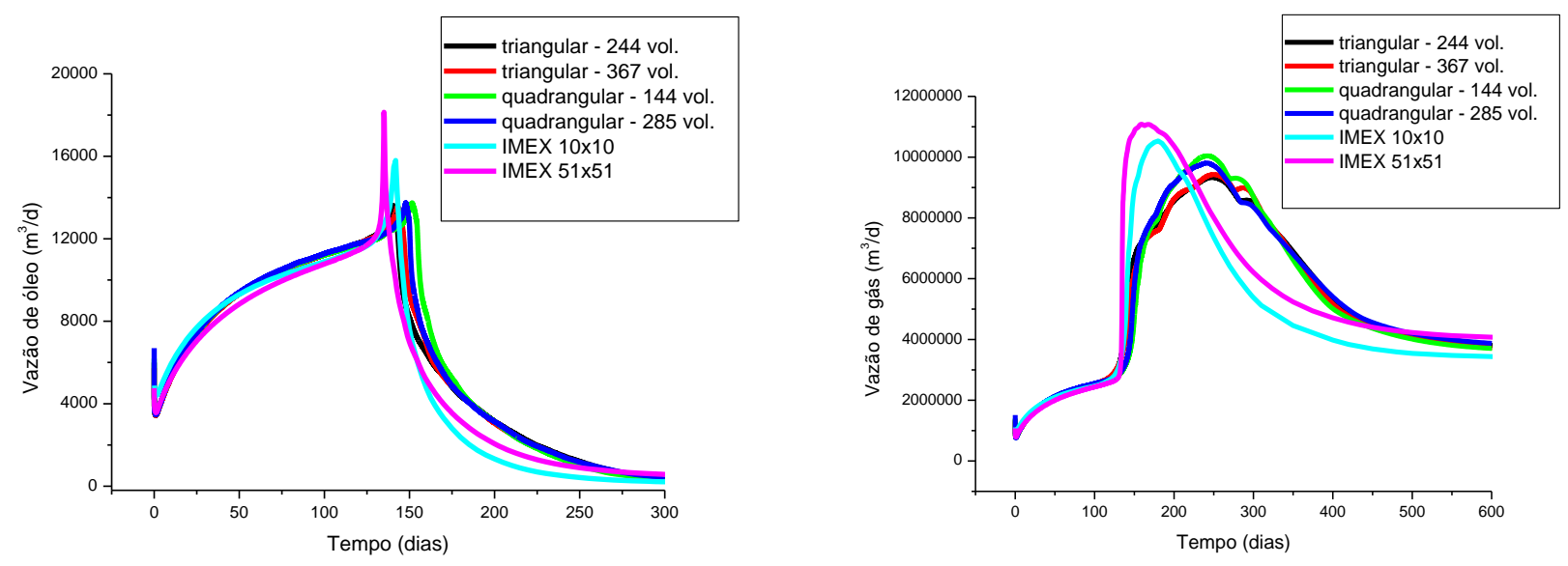

Figura 7 - (injeção de gás). Produção de óleo e gás. 

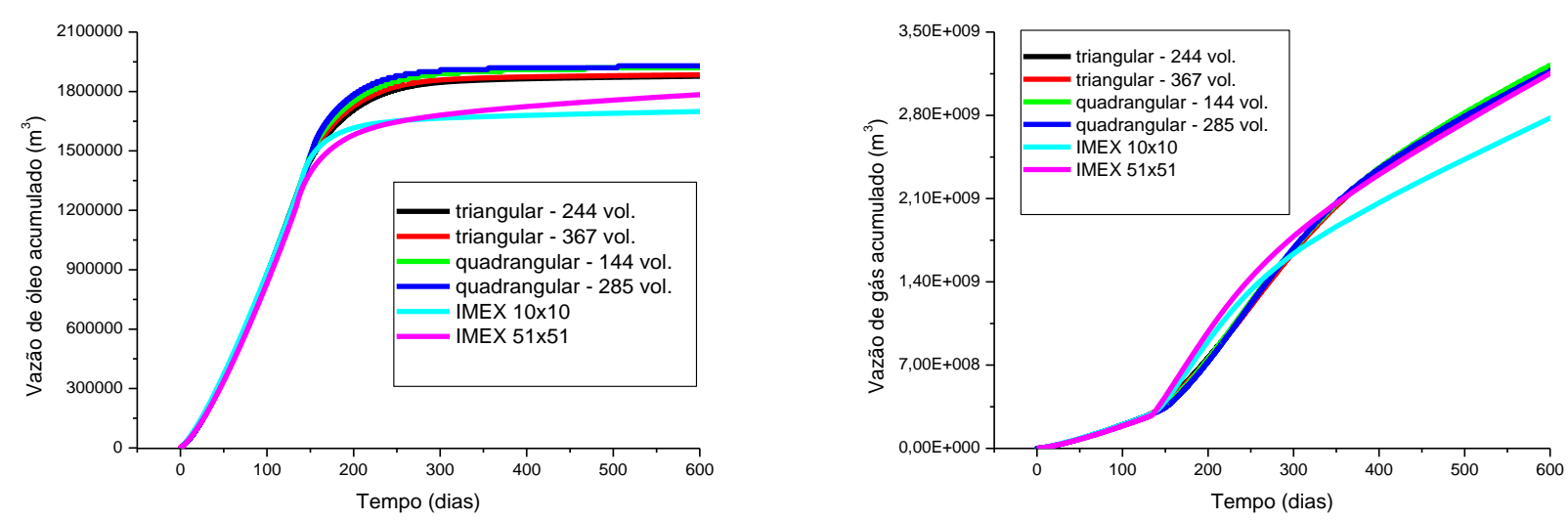

Figura 8 - (injeção de gás). Volume acumulado de óleo e gás.

As curvas de produção de óleo e água, injetando água ao invés de gás estão listadas na Figura 9. Desta figura pode-se constatar que os resultados da presente formulação apresentaram uma razoável concordância com os resultados obtidos com o simulador comercial. Destaca-se ainda que aproximadamente o mesmo tempo para a obtenção do breakthrough da água foi obtido em todas as simulações realizadas com os simuladores BLACK e IMEX.
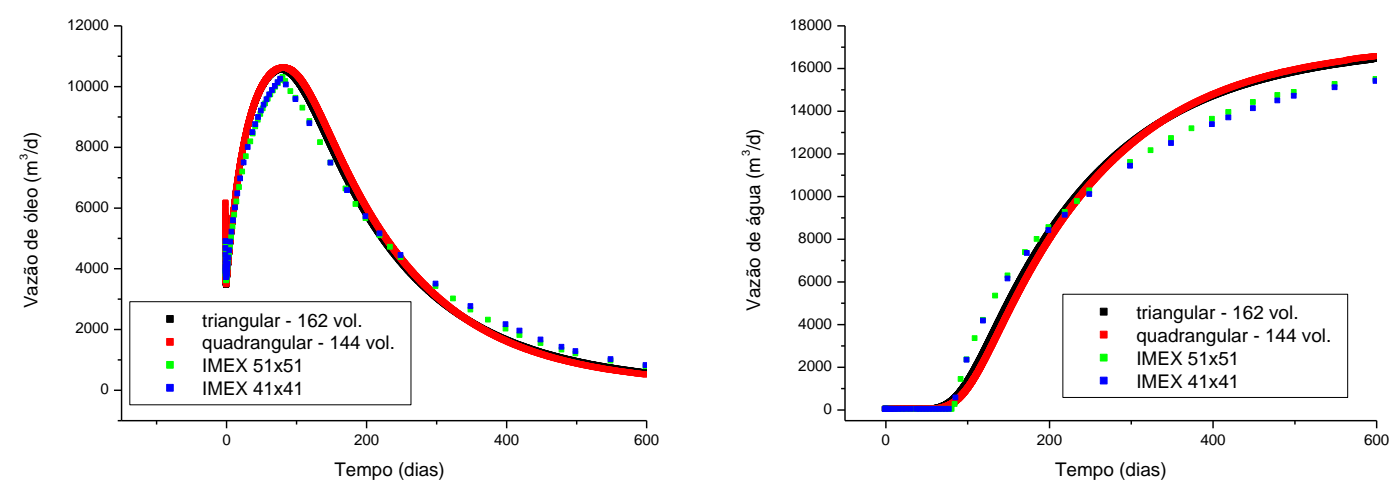

Figura 9 - injeção de água. Produção de óleo e gás.

O segundo caso novamente refere-se a um quarto de five-spot, porém, neste, é considerada a simulação de um reservatório anisotrópico. Para este caso, será considerada apenas a injeção de água. A malha será rotacionada em $45^{\circ}$ com o intuito de mostrar que a metodologia EbFVM apresenta um menor efeito de orientação de malha. Apesar dos resultados do problema físico serem completamente independentes da orientação da malha, sabe-se que os resultados numéricos apresentam uma elevada sensibilidade à rotação da malha.

Os campos de saturação de água para o caso 3 em 210 dias de produção considerando a injeção de água, estão listados nas Figs. 10-13. Destas figuras, pode ser constatado que as soluções obtidas com os elementos triangulares e quadrangulares com e sem rotação apresentaram uma razoável concordância. Este fato demonstra que a metodologia EbFVM, 
conforme discutido em Marcondes e Sepehrnoori (2010) apresenta menores efeitos de orientação de malhas.

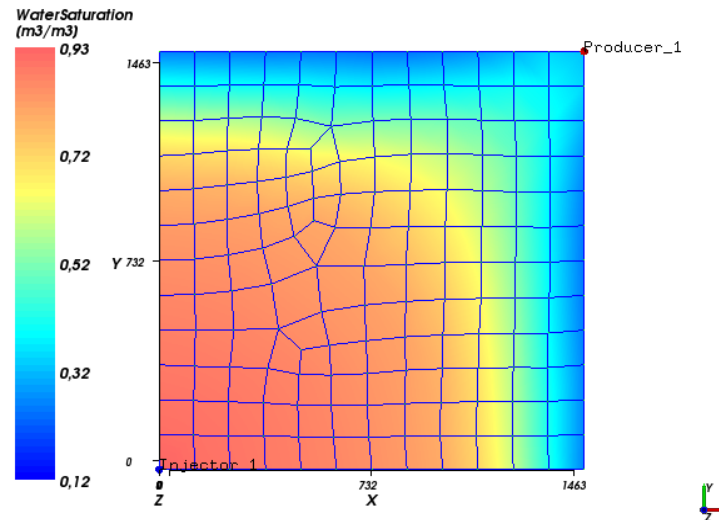

Figura 10 - Caso 2. Saturação de água - 210 dias - malha quadrangular.

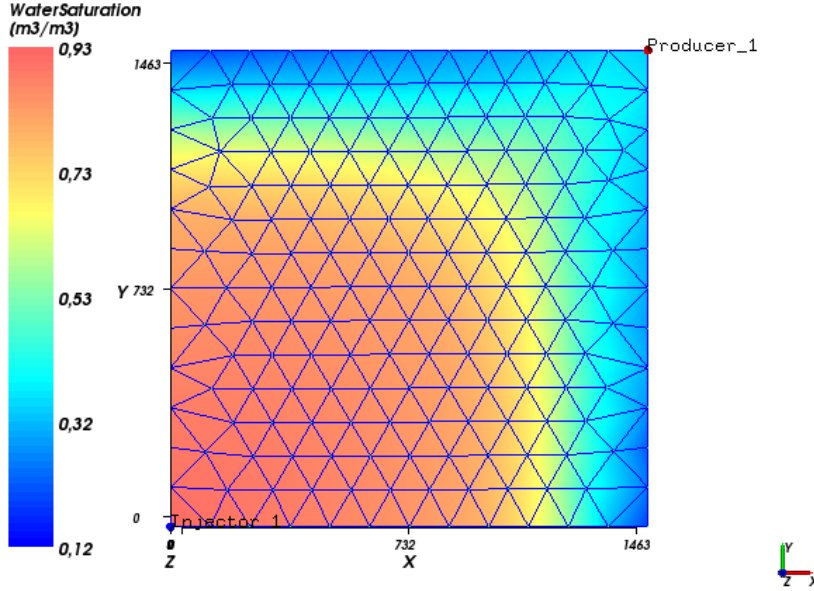

Figura 11 - Caso 2. Saturação de água - 210 dias - malha triangular.

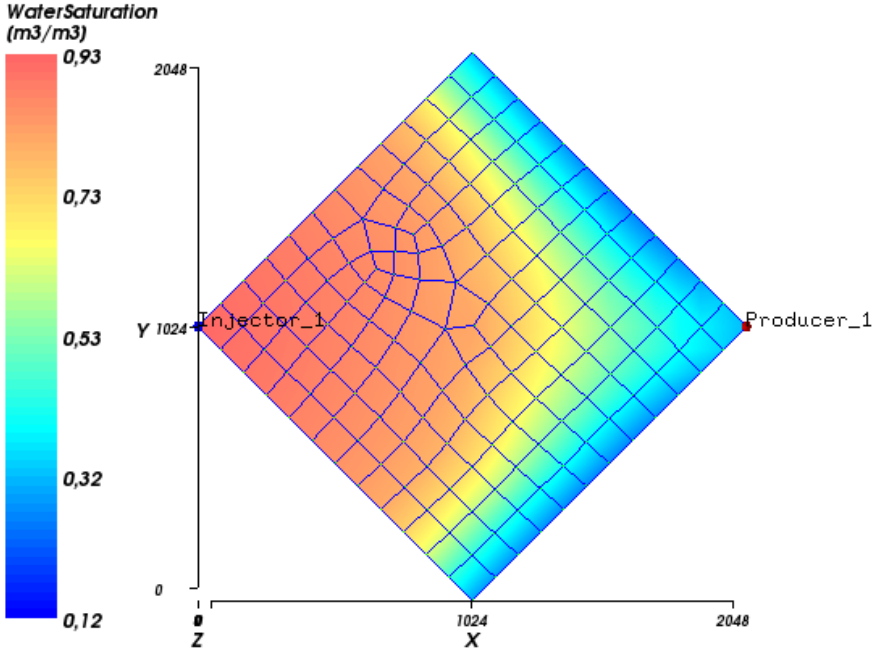

$\frac{1}{2 x}$ 
Figura 12 - Caso 2. Saturação de água - 210 dias - malha quadrangular rotacionada em $45^{\circ}$.

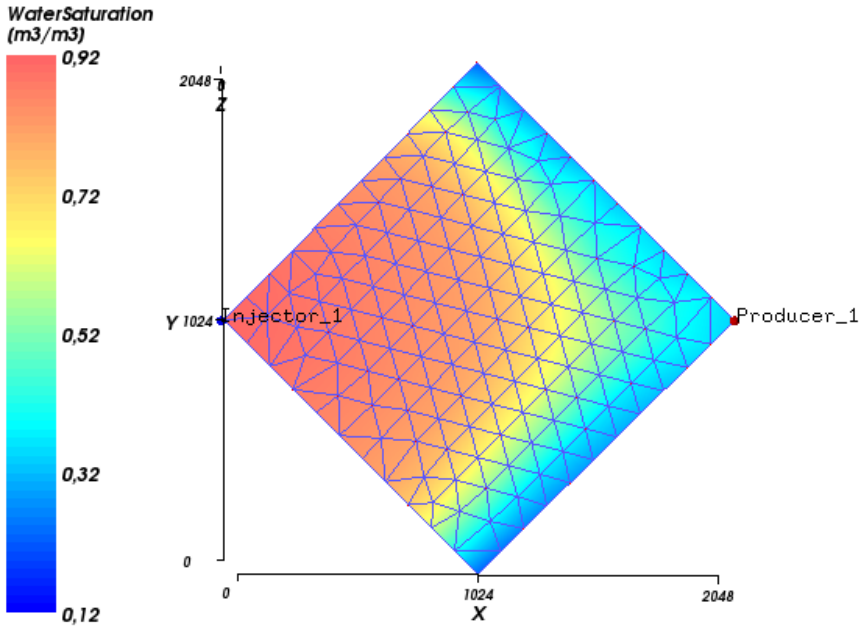

Figura 13 - Caso 2. Saturação de água - 210 dias - malha triangular rotacionada em $45^{\circ}$.

As curvas de produção de óleo e água para este caso estão apresentadas nas Figuras 14 e 15. Desta figura, pode-se observar que os resultados obtidos com as malhas de quadriláteros e triângulos sem rotação apresentam uma boa concordância com aqueles das malhas rotacionadas, demonstrando desta forma, um pequeno efeito de orientação de malha da metodologia EbFVM. Este fato era esperado, visto que os campos de saturação apresentados nas Figuras 10-13 apresentaram uma excelente concordância.

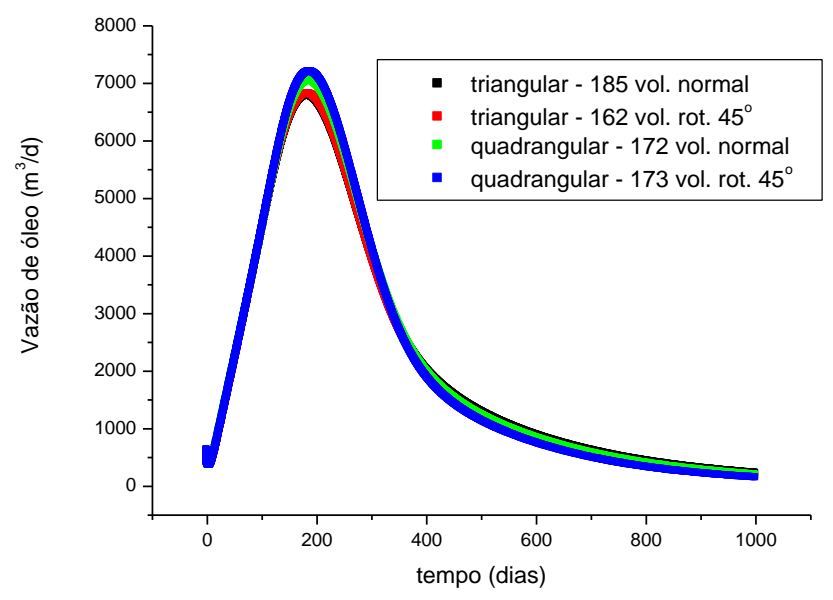

Figura 14 - Caso 2 (injeção de água). Produção de óleo. 


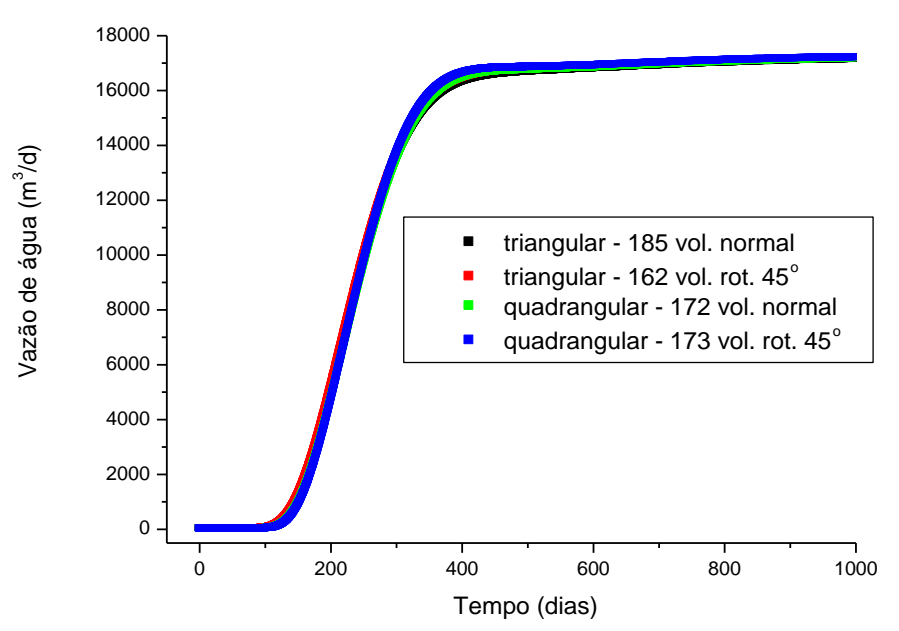

Figura 15 - Caso 2 (injeção de água). Produção de água.

\section{CONCLUSÕES}

Este trabalho apresentou uma abordagem baseada em elemento para o modelo blackoil em termos de pressão e fração mássica global em conjunto com malhas não estruturadas. Os resultados para a água e as saturações de gás, bem como recuperação primária foram comparados com o simulador comercial IMEX usando malhas cartesianas. Malhas não estruturada com elementos triangulares e quadrangulares foram utilizadas para testes e verificações da abordagem implementada. Os resultados em termos de taxas de volume de óleo e gás ficaram em boa concordância com os obtidos com malhas cartesianas.

Mostrou-se que o refinamento local é realizado de forma natural no método proposto, sem as dificuldades encontradas nos métodos que utilizam malhas estruturadas. Em malhas estruturadas um refino local de malha implica um aumento significativo do número de volumes em grande parte do domínio de cálculo, ao passo que nas malhas não estruturadas refina-se apenas localmente a região desejada.

Mostrou-se que os termos das equações denominados usualmente de transmissibilidades podem e precisam assumir valores negativos quando ângulos obtusos aparecem nos elementos triangulares da malha. A prática na área numérica, contudo, tem demonstrado que a presença de triângulos muito deformados não é uma boa conduta, mas a rejeição de uma malha pautada pela simples análise do sinal destes termos não se justifica.

Os resultados do método proposto mantiveram-se fisicamente coerentes mesmo em malhas formadas por elementos de razão de aspecto desfavorável.

\section{LISTA DE SÍMBOLOS}

$A=$ Área de reservatório $\left(\mathrm{m}^{2}\right)$

$B i-C G S T A B=$ Method of Bi-Conjugate Gradient Stabilized

$B_{p}=$ Fator formação de volume da fase $p\left(\mathrm{~m}^{3} / \mathrm{m}^{3}\right)$ 
$B_{w}=$ Fator formação de volume da fase água $\left(\mathrm{m}^{3} / \mathrm{m}^{3}\right)$

$B_{o}=$ Fator formação de volume da fase óleo $\left(\mathrm{m}^{3} / \mathrm{m}^{3}\right)$

$B_{g}=$ Fator formação de volume da fase gás $\left(\mathrm{m}^{3} / \mathrm{m}^{3}\right)$

$B_{p, \text { ref }}=$ Fator formação de volume referencia da fase $p\left(\mathrm{~m}^{3} / \mathrm{m}^{3}\right)$

$B_{p, \text { STC }}=$ Fator formação de volume Standard da fase $p\left(\mathrm{~m}^{3} / \mathrm{m}^{3}\right)$

$C M G=$ Computer Modelling Group Ltd.

$C_{r}=$ Compressibilidade da rocha $\left(\mathrm{Pa}^{-1}\right)$

$C_{o}=$ Compressibilidade de óleo $\left(\mathrm{Pa}^{-1}\right)$

$C_{w}=$ Compressibilidade de água $\left(\mathrm{Pa}^{-1}\right)$

$C_{p}=$ Compressibilidade da fase $p\left(\mathrm{~Pa}^{-1}\right)$

$E b F V M=$ Element based Finite Volume Method

$E O R=$ Enhanced Oil Recovery

ESSS = Engineering Simulation Scientific Software

$F_{p}=$ Fluxo mássica líquido da fase $p$ no volume de controle $\mathrm{P}$

$G_{i}=$ Fator geométrico

IMEX = Implicit-Explicit Black Oil Simulation

$J_{t}=$ Matriz Jacobiana

$K=$ Tensor permeabilidade absoluta

$k_{r p}=$ Permeabilidade relativa da fase $p$

$k_{\text {rog }}=$ Permeabilidade relativa da fase óleo-gás

$k_{\text {row }}=$ Permeabilidade de interface óleo água

$\dot{m}_{p}=$ Vazão mássica da fase $p$ por unidade de volume poroso do bloco contendo o poço $\left(\mathrm{kg} / \mathrm{m}^{3} \mathrm{~s}\right)$

$m_{i p}=$ Massa de componente $i$ da fase $\mathrm{p}(\mathrm{kg})$

$m_{p}=$ Massa da fase $p(\mathrm{~kg})$

$n_{c}=$ Número de componentes

$n_{p}=$ Número da fases

$N N E=$ Número de nós de um elemento da malha

$P=$ Pressão $(\mathrm{Pa})$

$P_{w f}=$ Pressão de fundo de poço $(\mathrm{Pa})$

$P_{\text {ref }}=$ Pressão de referência $(\mathrm{Pa})$

$P_{p}=$ Pressão da fase $p(\mathrm{~Pa})$

$q_{p}=$ Vazão da fase $p\left(\mathrm{~m}^{3} / \mathrm{s}\right)$

$r_{w}=$ Raio de poço $(m)$

$S_{L}=$ Saturação líquido (óleo + água) 
$S_{w}=$ Saturação de água

$S_{o i}=$ Saturação inicial de óleo

$S_{w i}=$ Saturação inicial de água

$S_{p}=$ Saturação da fase $p$

$U D S=$ Upstream difference scheme

$V_{\phi}=$ Volume poroso $\left(\mathrm{m}^{3}\right)$

$V_{t}=$ Volume total $\left(\mathrm{m}^{3}\right)$

$V_{v}=$ Volume dos vazios $\left(\mathrm{m}^{3}\right)$

$V_{s}=$ Volume do sólido $\left(\mathrm{m}^{3}\right)$

$V_{i p}=$ Volume de componente $i$ na fase $p\left(\mathrm{~m}^{3}\right)$

$V_{p}=$ Volume da fase $p\left(\mathrm{~m}^{3}\right)$

$X_{i p}=$ Fração mássica de componentes i na fase $p$

$X_{g g}=$ Frações mássica de componentes gás na fase gás

$X_{o o}=$ Frações mássica de componentes óleo na fase óleo

$X_{w w}=$ Frações mássica de componentes água na fase água

$x, y, z=$ Coordenadas cartesianas

$W I=$ Índice de poço $\left(\mathrm{m}^{3}\right)$

$Z_{i}=$ Fração mássica global de componentes $i$

$Z_{p}=$ Fração mássica de componente $p$

\section{Gregos}

$\alpha_{p}=$ Fração mássica de fase $p$

$\lambda_{p}=$ Mobilidade da fase $p$

$\phi=$ Porosidade

$\Phi_{p}=$ Potencial da fase $p(\mathrm{~Pa})$

$\xi=$ Coordenada generalizada na direção ksi

$\eta=$ Coordenada generalizada na direção eta

$\rho_{i}=$ Densidade de componente $i\left(\mathrm{~kg} / \mathrm{m}^{3}\right)$

$\rho_{m}=$ Densidade média $\left(\mathrm{kg} / \mathrm{m}^{3}\right)$

$\rho_{p}=$ Densidade da fase $p\left(\mathrm{~kg} / \mathrm{m}^{3}\right)$

$\rho_{p, S T C}=$ Densidade da fase $p$ nas condições padrão

$\rho_{r}=$ Densidade da rocha $\left(\mathrm{kg} / \mathrm{m}^{3}\right)$

$\mu_{p}=$ Viscosidade da fase $p(\mathrm{Cp})$ 


\section{REFERÊNCIAS BIBLIOGRÁFICAS}

1. ABOU-KASSEM, J.H. and AZIZ, K. "Handling of phase change in thermal simulators." SPEJ, September. 1985.

2. ARAÚJO, A. L. S., Solução de Escoamentos de Fronteira Livre Usando Malhas NãoEstruturadas e Volumes Finitos Baseados em Elementos Finitos, Tese de Doutorado, Departamento de Engenharia Mecânica, Universidade Federal da Paraíba, João Pessoa-PB, Março, 2004.

3. ARAÚJO, J. J. P., "Simulação Tridimensional de Reservatório de Petróleo Usando Modelo Black-Oil com as Formulações em Frações Mássicas", Dissertação de Mestrado, Departamento de Engenharia Química, Universidade Federal do Ceará, Fortaleza-CE, Novembro, 2005.

4. BALIGA, B.R. e PATANKAR, S.V., 1980, "A New Finite Element Formulation for Convection Diffusion Problems", Numerical Heat Transfer, Vol. 3, pp. 393-409.

5. BHARGAVA, D.S. Effects of environmental factors on nitrification during aerobic digestion of activated sludge. Journal of the Institution of Engineering (India), Part EN: Environmental Engineering Division, v.68, n.2, p.29-35, Feb. 1988.

6. CORDAZZO, J., 2004, An Element Based Conservative Scheme using Unstructured Grids for Reservoir Simulation, SPE International Student Paper Contest, The SPE Annual Technical Conference and Exhibition, Houston, Texas.

7. CORDAZZO, J., MALISKA, C. R., SILVA, A. F. C., and HURTADO, F. S. V., 2004, The Negative Transmissibility Issue When Using CVFEM in Petroleum Reservoir Simulation - 1. Theory", The 10th Brazilian Congress of Thermal Sciences and Engineering - ENCIT 2004, Braz. Soc. of Mechanical Sciences and Engineering - ABCM, Rio de Janeiro, Brazil, Nov. 29-Dec. 03.

8. COUTINHO, B. G., Simulação Numérica de Problemas de Reservatório de Petróleo Usando Coordenadas Generalizadas, Dissertação de Mestrado, Universidade Federal de Campina Grande-PB, Agosto, 2002.

9. CUNHA, A. R. C., "Uma Metodologia para Simulação Numérica Tridimensional de Reservatórios de Petróleo Utilizando o Modelo Black-Oil e Formulação nas Frações Mássicas", Dissertação de Mestrado, Departamento Eng. Mecânica, Universidade Federal de Santa Catarina, Maio, 1996.

10. FORSYTH, P.A. and SAMMON, P.H. "Gas phase appearance and disappearance in fully implicit Black Oil simulation." SPEJ, Oct. 1984.

11. HUGHES, T.J.R., The Finite Element Method, Linear Static and Dynamic Finite Element Analysis. Prentice Hall, New Jersey. 1987.

12. KARPINSK, L., MALISKA, C.R., MARCONDES, F. DELSHAD, M., and SEPHRNOORI, K., 2009, An Element Based Conservative Approach Using Unstructured Grids in Conjunction with a Chemical Fooding Compositional Reservoir Simulator, 20th International Congress of Mechanical Engineering, Gramado, RS.

13. MALISKA, C.R., da SILVA, A.F.C., CZESNAT, A.O., LUCIANETTI, R.M., and MALISKA Jr., C.R., 1997, "Three-Dimensional Multiphase Flow Simulation in Petroleum Reservoirs using the 
Mass Fractions as Dependent Variables", SPE 39067-MS, Latin American and Caribbean Petroleum Engineering Conference, Rio de Janeiro, Brazil.

14. MARCONDES, F., ZAMBALDI, M.C., MALISKA, C. R., Comparação de Métodos Iterativos não Estacionários em Malhas não Estruturas de Voronoi na Solução de Problemas de Petróleo, XII COBEM, Belo Horizonte, MG,1995.

15. MARCONDES, F., and SEPEHRNOORI, K., 2007, Unstructured Grids and an Element Based Conservative Approach for Compositional Reservoir Simulation, The 19th International Congress of Mechanical Engineering, November 5-9, Brasília, DF, Brazil.

16. MARCONDES, F., and SEPEHRNOORI, K., 2010, An Element-based Finite-Volume Method Approach for Heterogeneous and Anisotropic Compositional Reservoir Simulation, to appear in Journal of Petroleum Science and Engineering.

17. NOGUEIRA, R. L., FERNANDES, B. R. B., ARAÚJO, A. L. S., MARCONDES, F., Unstructured Grids And An Element Based Conservative Approach For A Black-Oil Reservoir Simulation, 2010, 13th Brazilian Congress of Thermal Sciences and Engineering, Dezembro, Uberlandia, MG, Brasil.

18. NOGUEIRA, R. L., "SIMULAÇÃO DE RESERVATÓRIO DE PETRÓLEO UTILIZANDO O MODELO BLACK-OIL BASEADO EM FRAÇÕES MÁSSICAS EM CONJUNTO COM MALHAS NÃO ESTRUTURADAS", Dissertação de Mestrado, Departamento Eng. Química, Universidade Federal Do Ceará , Fevereiro, 2011.

19. PRAIS, F. e CAMPAGNOLO, E. A., 1991, Modelagem do Escoamento Multifásico em Simulação de Reservatório, XI COBEM, São Paulo, SP, Dezembro.DATAR, M.T..

20. SARMENTO, D. C., Simulação trifásica de reservatórios de petróleo utilizando o modelo black-oil com formulação nas frações mássicas, Dissertação de Mestrado, Departamento de Engenharia Química, Universidade Federal do Ceará, Fortaleza-CE, Março, 2009.

21. VAN DER VORST, H. A. BI-CGSTAB, A fast and smoothly converging variant of Bi-CG for the simulation of nonsymmetrical linear systems, SIAM J. SCI. STAT COMPUT, March, 1992. 